\title{
Out of darkness comes light-is there a role for light masks in treatment of diabetic macular oedema?
}

\author{
Mali Okada ${ }^{1}$, Jay Chhablani \\ ${ }^{1}$ Department of Ophthalmology, Royal Victorian Eye and Ear Hospital, Melbourne, Australia; ${ }^{2}$ Smt Kanuri Santhamma Center for Vitreoretinal \\ Diseases, LV Prasad Eye Institute, Hyderabad, India \\ Correspondence to: Jay Chhablani. Smt Kanuri Santhamma Center for Vitreoretinal Diseases, LV Prasad Eye Institute, Hyderabad, Andhra Pradesh, \\ India. Email: jay.chhablani@gmail.com. \\ Provenance: This is an invited Editorial commissioned by the Section Editor Qi-Nan Wu (Chongqing University Cancer Hospital \& Chongqing \\ Cancer Institute \& Chongqing Cancer Hospital, Chongqing, China). \\ Comment on: Sivaprasad S, Vasconcelos JC, Prevost AT, et al. Clinical efficacy and safety of a light mask for prevention of dark adaptation in treating \\ and preventing progression of early diabetic macular oedema at 24 months (CLEOPATRA): a multicentre, phase 3, randomised controlled trial. \\ Lancet Diabetes Endocrinol 2018;6:382-91.
}

Submitted Oct 15, 2018. Accepted for publication Oct 18, 2018.

doi: $10.21037 / \mathrm{atm} .2018 .10 .50$

View this article at: http://dx.doi.org/10.21037/atm.2018.10.50

\section{Introduction}

Diabetic macular edema (DME) is a significant cause of visual impairment in working aged adults, with an estimated global prevalence of $7 \%$ of all diabetic patients (1). Its relative importance continues to rise, with vision loss from DME now surpassing proliferative diabetic retinopathy (PDR) as the leading cause of vision impairment from diabetes in developing countries (2).

The treatment paradigm for DME has evolved significantly over the years. The Early Treatment in Diabetic Retinopathy study in 1985 established focal/grid macular laser as the mainstay of treatment for more than two decades (3). However, although macular laser reduced moderate vision loss in up to $50 \%$ of patients, visual gain was less reliable. More recently, intravitreal injection therapies, including anti-vascular endothelial growth factor (anti-VEGF) or intraocular steroids, has become the standard of care for center-involving DME, with several large randomized controlled trials demonstrating consistent visual gains and superior outcomes for intravitreal injections as compared to traditional macular laser $(4,5)$.

However, intravitreal injection therapy for DME is not without its limitations. Frequent injections are required, with an average of 15 injections needed over 2 years to maintain vision (6). This treatment burden exerts significant strain not only on patients but also on healthcare systems worldwide. Intravitreal injections are also invasive, and although serious complications are rare, risks include post-injection endophthalmitis, which can be devastating. Therefore, the search for other strategies to combat DME continues.

\section{Rod energy consumption}

One novel therapeutic approach has focused on the unique property of the retina during the state of dark adaptation. Although retinal tissue shares a similar origin and features with the rest of the central nervous system, it is differentiated by the presence of photoreceptors cells. The process of signal phototransduction by photoreceptors is energy intensive and account for the retina as the highest oxygen consuming tissue in the body. During darkness, energy demand by rods is at its maximum, resulting in reduced oxygen tension and relative retinal hypoxia (7). This hypoxic condition occurs in healthy eyes but is compounded in diseased states such as diabetic retinopathy, where there is relative impairment of oxygen supply, even in early stages (8). Retinal hypoxia is a potent stimulus for VEGF, the upregulation of which contributes to increased vascular permeability and worsening of DME. 


\section{Light masks}

Light mask therapy is based on the premise that preventing the dark state reduces the oxygen demands on the eye, and over time, this may slow or reduce diabetic retinopathy and DME progression. Preliminary studies using an illuminated patch over one eye during sleep reported improved colour contrast threshold, reduced microaneurysms and retinal haemorrhages as well as reduced retinal thickness compared to fellow eyes $(9,10)$. However, these were small studies of short duration only, and lacked an adequately controlled arm.

Recently, a large multicenter randomized controlled trial (CLEOPATRA study) compared the use of light mask versus sham mask during sleep in patients with noncenter involving DME (11). This UK study utilized eye masks (Noctura 400 Sleep Mask, PolyPhotonic Medical) with blue-green emitting LED lights and the primary outcome was change in maximal retinal thickness of optical coherence tomography (OCT) at 24 months. Compliance was assessed with the use of in-built recorders that detect when the device is in use. The study enrolled 308 patients with a total 246 patients contributing anatomical data at the 24 months time point.

Disappointingly, the results of the study revealed that there was no difference in the change in retinal thickness between the light masks versus sham masks groups (adjusted mean difference of $-0.65 \mathrm{~m}, \mathrm{P}=0.84$ ). Other outcome measures assessed, including development of center-involving DME, need for additional therapy and progression of diabetic retinopathy, were also not different between the treatment groups.

Although the study was well designed, the major limitation of the study also reflects the greatest inherent weakness of this form of therapy, that of poor patient compliance. Even early on, compliance at 4 months was only at $39.5 \%$, dropping to an abysmal $19.5 \%$ by 24 months. Considering that this was in the context of a clinical trial, where patient cooperation and motivation tends to be higher, translating these figures to the real world would likely result in even lower numbers. Causes for this may be multifactorial. Surprisingly only 7 out of 155 patients in the active treatment group reported sleep disturbance during the trial. The majority of the other adverse effects reported appeared to relate to the use of the mask itself. Even in the sham mask group, compliance was poor, with the study investigators deciding early on during the trial to allow patients allocated to the sham group the option of discontinuing to wear the sham mask as very few were wearing it. It is therefore possible, that non-adherence in the treatment group could have resulted in an underestimation of the effect from the light mask in this study. However, assessment of results at different timepoints in the study did not reveal any significant difference, even at 4 months when compliance was highest.

The authors also hypothesized that the negative result for this study could potentially be due to insufficient light intensity from the mask to alter the rod dark current state. Whilst this may be true, it is difficult to see how increasing the retinal illumination could be achieved in this setting, without also disrupting sleep and further reducing compliance.

The lack of a treatment effect from light masks in this study is in contrast to previous reports by the same authors. One possibility for this includes the differences in control groups used, with both preliminary studies only light patching one eye and utilizing the fellow eye as the control group. Small number of patients and variable follow up may also be responsible. Studies such as the CLEOPATRA trial therefore reinforce the importance for well-designed studies to ensure results are valid and can be replicated.

So where does this leave the concept of light therapy in management of retinovascular diseases? Would changing the wavelength improve efficacy and tolerability? Perhaps other methods of light delivery such as phototherapy or even light emitting electrode implants could even be considered. Based on its current format as a light mask however, long term efficacy and feasibility for this approach remains doubtful. Further light mask trials currently on the horizon for patients with center-involving refractory DME may prove otherwise (12). Nevertheless there remains sufficient biological plausibility to pursue further investigation into dark suppression as a method of reducing the hypoxic drive in diabetic eye disease. However, a rethink of the format and delivery in order to achieve this will be required.

\section{Acknowledgements}

None.

\section{Footnote}

Conflicts of Interest: M Okada and J Chhablani received travel and honoraria from Allergan. 


\section{References}

1. Yau JW, Rogers SL, Kawasaki R, et al. Global prevalence and major risk factors of diabetic retinopathy. Diabetes Care 2012;35:556-64.

2. Zhang X, Saaddine JB, Chou CF, et al. Prevalence of diabetic retinopathy in the United States, 2005-2008. JAMA 2010;304:649-56

3. Photocoagulation for diabetic macular edema. Early Treatment Diabetic Retinopathy Study report number 1. Early Treatment Diabetic Retinopathy Study research group. Arch Ophthalmol 1985;103:1796-806.

4. Diabetic Retinopathy Clinical Research Network, Elman MJ, Aiello LP, et al. Randomized trial evaluating ranibizumab plus prompt or deferred laser or triamcinolone plus prompt laser for diabetic macular edema. Ophthalmology 2010;117:1064-77.e35.

5. Virgili G, Parravano M, Evans JR, et al. Anti-vascular endothelial growth factor for diabetic macular oedema: a network meta-analysis. Cochrane Database Syst Rev 2017;6:CD007419.

6. Wells JA, Glassman AR, Ayala AR, et al. Aflibercept, Bevacizumab, or Ranibizumab for Diabetic Macular Edema: Two-Year Results from a Comparative Effectiveness Randomized Clinical Trial. Ophthalmology

Cite this article as: Okada $M$, Chhablani J. Out of darkness comes light-is there a role for light masks in treatment of diabetic macular oedema? Ann Transl Med 2018;6(Suppl 1):S73. doi: $10.21037 / \mathrm{atm} .2018 .10 .50$ 